\title{
MÁS DE TREINTA AÑOS DE DROGAS ILEGALES EN ESPAÑA: UNA AMARGA HISTORIA CON ALGUNOS CONSEJOS PARA EL FUTURO (*)
}

\author{
Luis de la Fuente (1,2), M Teresa Brugal (3), Antonia Domingo-Salvany (4), María J Bravo (5), \\ Montserrat Neira-León (2) y Gregorio Barrio (5) \\ (1) Centro Nacional de Epidemiología. Instituto de Salud Carlos III. Madrid. \\ (2) Secretaría del Plan Nacional sobre el Sida. Ministerio de Sanidad y Consumo. Madrid. \\ (3) Agència de Salut Pública de Barcelona. \\ (4) Unidad de Investigación en Servicios Sanitarios. Institut Municipal d'Investigació Mèdica (IMIM). Barcelona. \\ (5) Departamento de Medicina Preventiva, Salud Pública e Historia de la Ciencia. Universidad Complutense de Madrid. \\ (*) Financiación: Ciber de Epidemiología y Salud Pública. Instituto de Salud Carlos III, 2006.
}

\section{RESUMEN}

Las dramáticas consecuencias del consumo de heroína (principalmente inyectada) han marcado el fenómeno de las drogas ilegales en España en los últimos treinta años. Más de 300.000 personas han sido tratadas por dependencia de heroína, 20.000-25.000 han muerto por «sobredosis», 100.000 han adquirido el VIH mediante inyección de drogas y bastantes más se han infectado con los virus de la hepatitis. Algunas de estas consecuencias pueden atribuirse al retraso en la puesta en marcha de intervenciones efectivas, como los tratamientos de mantenimiento con metadona (TMM). Actualmente han descendido mucho estos problemas por el descenso del número de personas que se inyectan y el efecto de las intervenciones, principalmente los TMM. Sin embargo, la mortalidad por sobredosis sigue siendo muy alta (más de 700 muertes anuales), y entre los consumidores por vía intravenosa persiste una elevada prevalencia de VIH y hepatitis C, y están emergiendo las consecuencias de las hepatopatías crónicas. Paralelamente, ha aumentado espectacularmente el uso de la cocaína y con él los problemas que causa: hay más de 100.000 consumidores semanales, 25.000 personas tratadas anualmente por abuso o dependencia, y un impacto importante sobre los servicios médicos de urgencia. Su efecto sobre la mortalidad es desconocido. Están aumentando también el consumo y los problemas por cannabis (existe medio millón de consumidores diarios). Se propone mantener y reforzar los programas de reducción del daño (TMM, intercambio de jeringas, salas de consumo, vacunación de hepatitis A y B, etc.), desarrollar con urgencia estrategias específicas para reducir las muertes por sobredosis y los problemas por cocaína, y reevaluar la efectividad de las estrategias preventivas y de control de la oferta.

Palabras clave: Drogas ilegales. Cannabis. Cocaina. Heroina. VIH. Hepatitis. España. Metadona.

Correspondencia:

Luis de la Fuente

Centro Nacional de Epidemiología. Instituto de Salud Carlos III

C/Sinesio Delgado 6

28029 Madrid

Correo electrónico:Ifuente@isciii.es

\section{ABSTRACT
More than thirty years of illicit drugs in Spain: a bitter story with some messages for the future

The phenomenon of illicit drug use in Spain during the last thirty years has been marked by the extremely serious consequences of heroin use (mainly injecting). More than 300.000 persons were treated for heroin dependence, 20.000-25.000 died from overdose, 100.000 became infected with HIV through drug injection and quite more with hepatitis virus. Some of these consequences can be attributed to the delay in the implementation of effective interventions, such as methadone maintenance treatment (MMT). Currently, the decreasing number of injectors and the positive effects of interventions, mainly MMT, have led to an important decline of the mentioned health problems. However, overdose mortality remains very high (more than 700 deceased per year), prevalence of both HIV and HCV are still high among injectors, and consequences of chronic liver diseases are emerging. In the last years the use of cocaine and associated problems have increased a lot. Nowadays there are more than 100.000 weekly cocaine users, 25.000 persons are annually treated from cocaine abuse or dependence, and cocaine has an important impact on medical emergency services, while its impact on mortality is unknown. Both cannabis use and related problems are increasing too (there are half a million of daily users). We propose to maintain and to strengthen harm reduction programs (MMT, syringe exchange, save-use and injection rooms, hepatitis A and B vaccination, etc.), to urgently develop specific strategies targeted to reduce overdose mortality and cocaine related problems, and to re-evaluate the effectiveness of preventive and supply control strategies.

Key words: Addiction, Opiate. Heroin. Cannabis. Cocaine. Illicit Drugs. Abuse Drugs. Hepatitis. HIV. Spain. Epidemiology. Methadone. 


\section{INTRODUCCIÓN}

En España el uso de drogas ilegales por grupos amplios de población es bastante reciente. Además, hasta el inicio del uso inyectado de heroína a finales de los setenta sus repercusiones sociosanitarias fueron aparentemente irrelevantes, y no hay apenas referencias de problemas importantes asociados al uso de alucinógenos o cannabis durante la transición política; ni con el uso de especialidades farmacéuticas con anfetaminas para preparar exámenes, evitar la fatiga o adelgazar ${ }^{1}$.

Aunque en el tabaco o el alcohol, por separado, causan más muertes y sufrimiento que todas las drogas ilegales juntas, este trabajo se centra sólo en estas últimas, incluyendo su uso concurrente con psicofármacos o drogas psicoactivas de comercio legal. Por otra parte, aunque las drogas ilegales tienen implicaciones en muchos órdenes de la vida personal y social, aquí se considera sobre todo su impacto en la salud pública, deteniéndose en las crisis que han representado o representan amenazas graves para la vida o la salud de la población. Apenas se mencionan, en cambio, los fenómenos de consumo cuyo impacto sanitario no ha sido hasta ahora importante. Finalmente, se plantean algunas estrategias e intervenciones que podrían ser razonables para disminuir el impacto sanitario de las drogas ilegales.

\section{EL LARGO RASTRO DE LA «EPIDEMIA DE HEROÍNA»}

A lo largo de los ochenta y principios de los noventa la «epidemia» del consumo de heroína en forma de inyección ocasionó en España un gran aumento de la mortalidad juvenil, la demanda de atención sanitaria, y la delincuencia contra la propiedad ${ }^{1}$, generando intensa alarma social, y siendo la responsable de que «las drogas» llegaran a percibirse como uno de los tres principales problemas de la población, junto el paro y el terrorismo. A ello contribuyó, sin duda, la rápida expansión de la infección por el virus de la inmunodeficiencia humana (VIH) asociada a inyección de drogas (que en España ha sido, y continúa siendo, sinónimo de inyección de heroína). Aunque la máxima incidencia de uso problemático de heroína se alcanzó seguramente en la primera mitad de los ochenta ${ }^{2}$, el mayor impacto y visibilidad de la epidemia se produjo a principios de los noventa. La mortalidad relacionada con las drogas alcanzó entonces su punto álgido (figura 1), llegando a ser la primera causa de muerte entre los jóvenes de las grandes ciudades $^{3-5}$. A partir de los datos publicados ${ }^{6-8}$ se estima que en España el mayor impacto de la mortalidad por sobredosis se produjo en 1991-1992 con más de 1.700 muertes anuales $(11,5$ muertes por cada 100.000 jóvenes de 15-39 años, $10,1 \%$ de todas las muertes de esas edades), en más del $90 \%$ de las cuales estaba implicada la inyección de heroína. Los nuevos diagnósticos de sida ligados a inyección de drogas alcanzaron su máximo en 1993-1995 con más de 3.500 casos anuales, y la mortalidad por VIH en 1995-1996 con casi 4.300 muertes anuales $(27,4$ por cada 100.000 jóvenes de 15-39 años, 25,3\% de todas las muertes en esas edades). Esto no debe confundir, ya que el VIH se había adquirido 6-11 años atrás. De hecho, la máxima incidencia de VIH ligado a inyección de drogas se produjo probablemente entre 1985 y $1987^{9}$, con aproximadamente 14.500 infecciones anuales (figura 1).

Sin embargo, la respuestas para aminorar y controlar los problemas no llegaron pronto ni fueron siempre las más adecuadas. Hasta 1985 no se creó el Plan Nacional sobre Drogas (PND), que contribuyó a estructurar una red de atención a estos problemas. Por su parte, los tratamientos de mantenimiento con metadona (TMM), una de las intervenciones más efectivas para disminuir las repercusiones del uso de heroína (mortalidad, infecciones, problemas sociales), fueron fuertemente restringidos por una norma legal en 1985 y sólo se desarrollaron amplia- 
Figura 1

Evolución de la infección por VIH en personas usuarias de drogas por vía intravenosa y de las muertes directamente relacionadas con drogas ilegales (DRDI o sobredosis) en España, 1980-2005

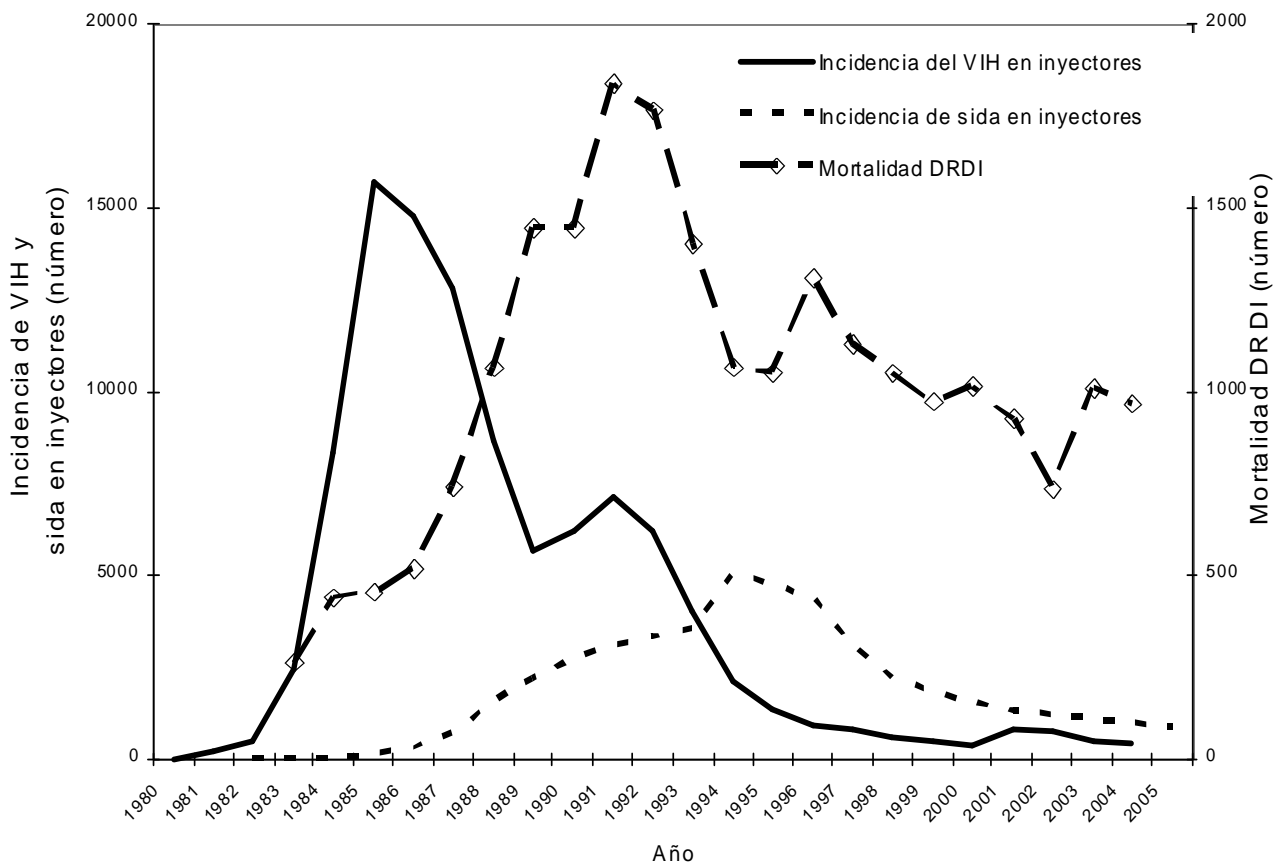

Incidencia de VIH: Número de nuevos casos de infección por VIH (incidencia) en inyectores de drogas en el conjunto de España. Los datos se obtuvieron como sigue:

- 1983-2000: Se hizo una estimación siguiendo la metodología utilizada por Castilla et al9 .

- 2001-2004: Se extrapolaron los datos del número de infecciones VIH en inyectores notificadas por ocho comunidades autónomas ${ }^{22}$ al conjunto de España.

Incidencia de Sida: Número de nuevos casos de sida ligados a inyección de drogas diagnosticados anualmente obtenidos del Registro Nacional de Sida, corregidos por retraso en la notificación ${ }^{88}$.

Mortalidad DRDI: Número de muertes directamente relacionadas con el consumo de drogas ilegales en el conjunto de España. Los datos se obtuvieron como sigue:

- 1983-1993: extrapolación de los datos de 6 ciudades (Madrid, Barcelona, Valencia, Sevilla, Zaragoza y Bilbao) obtenidos del Indicador Mortalidad del Observatorio Español sobre Drogas $(O E D)^{8 ; 36}$ al conjunto de España, asumiendo la hipótesis de que ambas áreas tienen la misma tasa de mortalidad.

- 1994-2001: estimación realizada por el OED ${ }^{8}$ basándose en Brugal MT et al ${ }^{7}$. Esta estimación asume que el nivel de subrregistro de estas muertes en el Registro General de Mortalidad en varias áreas conocidas es similar en el resto de España.

- 2002-2004: extrapolación a partir de datos publicados para algunas áreas ${ }^{37-39}$, asumiendo la hipótesis de que las áreas con datos desconocidos tiene la misma tasa de mortalidad que el conjunto de áreas cuyos datos se conocen.

mente, aunque de forma desigual según las Comunidades Autónomas (que desde 1990 tenían plenas competencias para hacerlo) después de 1992, cuando lo peor ya había pasado $^{10}$, y tras una intensa batalla frente a sus múltiples detractores de la sociedad civil y de los servicios de prevención y atención a las drogodependencias (algunos convertidos luego felizmente en gestores de los mismos). Aquel año comenzaron a disminuir progresivamente las muertes por sobredosis y poco después los diagnósticos de sida en usuarios 
de drogas por vía intravenosa aunque, como puede apreciarse, la incidencia de VIH ligada a inyección había comenzado a disminuir mucho antes (figura 1). Es muy probable que los TMM y los programas de intercambio de jeringas (PIJ), que se extendieron sobre todo a partir de mediados de los noventa, reforzaran la evolución positiva de estos problemas, pero todo apunta a que el principal determinante de la misma fue la fuerte disminución de la inyección de heroína (sustituida en parte por fumar heroína «en chinos»), producto más de cambios culturales o de mercado que de políticas públicas concretas ${ }^{10-13}$. Probablemente las políticas de reducción de daño indujeron o reforzaron el abandono de la inyección, pero no cabe duda de que su impacto estuvo muy mediatizado por la sustitución en gran parte del país de la heroína blanca por heroína marrón apta para fumar (en forma de base), por la percepción directa por parte de los consumidores de las consecuencias de la inyección, especialmente el sida, y por otros cambios culturales.

Actualmente el número de usuarios de heroína ha disminuido. Además, la mayoría no se inyectan $(63,7 \%$ de los admitidos a tratamiento en Cataluña en 2004 y 90,8\% en Andalucía en 2005) y muchos no se han inyectado nunca $(34,4 \%$ de los admitidos a tratamiento en Cataluña en 2004 y 54,4\% en Andalucía en 2005) ${ }^{14-15}$. Sin embargo, en muchas áreas, sobre todo en el nordeste de la península y Baleares, persisten grupos amplios de inyectores esporádicos o regulares $^{8}$. Además, en prisión se concentran muchos de ellos. Así, unos 7.000 internados prisión en 2006 (un 11,4\% del total) se habían inyectado drogas en los 30 días previos al último ingreso ${ }^{16}$.

Buena parte de los usuarios de heroína están en TMM, lo que ha contribuido a aliviar sus problemas y a disminuir bastante su riesgo de muerte. De hecho, se ha estimado que en Barcelona el $86 \%$ de las muertes por sobredosis y el $38 \%$ por VIH se hubieran evitado si los afectados hubieran estado en
$\mathrm{TMM}^{17}$. Sin embargo, los TMM ven disminuida su efectividad porque actualmente la mayoría de los usuarios de heroína son también dependientes de cocaína ${ }^{18}$, sustancia que no se beneficia de la acción sustitutiva de la metadona, y para la que no existe ningún tratamiento específico de efectividad bien comprobada ${ }^{19}$.

Las nuevas infecciones por VIH en usuarios de drogas por vía intravenosa han disminuido drásticamente, siendo superadas claramente por las infecciones a través de relaciones sexuales ${ }^{20-22}$. Sin embargo, los pocos estudios de seroconversión al VIH de usuarios de drogas por vía intravenosa con evidencias de una prueba reciente negativa muestran que siguen infectándose a un ritmo importante (4,5 por 100 personas-año -pa-, IC95\%: 2,9-6,7, en un trabajo muy reciente en jóvenes inyectores del Proyecto Itine$r e)^{23}$, lo que sugiere de nuevo que la caída del VIH ligado a inyección se ha debido sobre todo al descenso del número de usuarios de drogas por vía intravenosa. Hasta ahora se creía que la infección por VIH en usuarios de heroína que no se inyectan era poco frecuente, pero los últimos datos de Itinere muestran una incidencia muy alta en los jóvenes usuarios de heroína de Sevilla $(3,4 / 100 \text { pa; IC95\%: 0,9-8,7 })^{23}$. Esto confiere importancia a la transmisión sexual, y a la necesidad de prestar más atención a las parejas de sujetos seropositivos y de usuarios de heroína por vía intravenosa. Por otra parte, la vida de los infectados se ha alargado mucho con los nuevos tratamientos antirretrovirales, por lo que su prevalencia de VIH disminuirá despacio. De hecho, en España las personas con antecedentes de inyección de drogas siguen teniendo una prevalencia muy alta de infección por VIH (20\%-40\%) y de conductas de riesgo $8,16,21,24$.

En España la prevalencia de infección por los Virus de la hepatitis B (VHB) y C (VHC) entre los inyectores de drogas ha sido siempre muy alta. Así, en 2001-2003 era de 20-35\% para VHB y de $65 \%-86 \%$ para $\mathrm{VHC}^{25-28}$. 
Estas infecciones a menudo generan hepatopatías crónicas graves cuyas consecuencias en España han estado eclipsadas por la morbimortalidad causada por el VIH, pero que ahora están emergiendo nítidamente debido al descenso de la misma por los tratamientos antirretrovirales ${ }^{28-31}$. De hecho, en ciertos grupos de usuarios de heroína la mortalidad por hepatopatías puede ser ya casi tan alta como por $\mathrm{VIH}^{17}$. Además, en estos grupos suele hallarse un alto nivel de coinfección con VHC y VIH ${ }^{32}$, lo que aumenta la progresión de la hepatopatía y la mortalidad ${ }^{31}$. En los próximos años probablemente aumentará la demanda sanitaria por hepatopatías crónicas por VHC debido al diagnóstico de infecciones asintomáticas y a la descompensación de cirrosis no diagnosticadas, lo que acarreará un coste importante para el sistema sanitario, ya que el interferón y la ribavirina para un año de tratamiento de VHC cuestan alrededor de $16.000 €{ }^{33}$.

A pesar de que en España ha descendido la incidencia global de infección por VHC, probablemente debido al descenso de la inyección de drogas ${ }^{33}$ continúan las dificultades para controlar el VHC, porque entre los usuarios de la vía intravenosa persiste una alta prevalencia de infección y de prácticas de riesgo, sobre todo la de compartir materiales de inyección usados distintos de las agujas, como recipientes o jeringas para disolver o repartir la droga, filtros, líquido para limpiar las jeringas, etc. ${ }^{28,34,35}$. En la misma línea, los datos de Itinere muestran que la incidencia de VHC entre los jóvenes usuarios de la vía intravenosa continúa siendo altísima (34,8/100 pa; IC 95\%: 26-46) 23.

$\mathrm{Al}$ hacer un balance provisional de la epidemia de heroína las cifras resultan escalofriantes. Con los datos publicados ${ }^{7-9,22.36-39}$ se estima que unas 212.000 personas han sido tratadas por dependencia de esta droga en centros que notifican al indicador tratamiento del PND, por lo que los usuarios problemáticos deben haber sido más de 300.000. Unos 100.000 inyectores de drogas (prácticamente todos inyectores de heroína) se han infectado por VIH (figura 1), y bastantes más por VHC o VHB. Finalmente, se han producido entre 20.000 y 25.000 muertes por sobredosis o reacción aguda a drogas (figura 1), en más del $90 \%$ de los casos con implicación de heroína.

\section{NUEVOS RETABLOS, NUEVOS CANTARES}

Como se ha indicado, a partir de la segunda mitad de los noventa las consecuencias más graves de la epidemia del uso de heroína disminuyeron (mortalidad aguda, infección por VIH, delincuencia contra la propiedad) y las drogas ilegales dejaron de ser percibidas por los españoles como uno de sus principales problemas, por lo que la atención política y mediática se desvió hacia el uso de drogas en el contexto recreativo, principalmente éxtasis y cannabis ${ }^{40-42}$. Tanta atención se ha justificado diciendo que pueden ser el camino hacia drogas más peligrosas (léase cocaína o heroína), y que las alteraciones cerebrales que generan acabarán provocando a los consumidores problemas clínicos o sociales con un impacto importante en la salud pública, como ha sucedido con la cocaína. Estos argumentos están lejos de ser demostrados de forma satisfactoria, pero se han utilizado profusamente.

Más allá de las representaciones mediáticas o políticas, las evidencias sobre los problemas de drogas en España muestran lo siguiente: que persiste un núcleo duro y menguante de consumidores muy problemáticos de heroína que corre el riesgo de ser desatendido; que hay problemas importantes y crecientes ligados al uso de cocaína, que requieren una atención creciente; y que el cannabis y los psicoestimulantes sintéticos tipo éxtasis, a pesar de su extensión entre la población, no representan hoy por hoy un importante problema de salud pública en España, aunque ello no significa que no se 
asocien a consecuencias adversas de distinto tipo (incluidos problemas escolares) y de distinto nivel de gravedad en un buen número de consumidores, sobre todo en aquéllos que las consumen de forma intensiva o junto a otras drogas, como cocaína o alcohol.

Detengámonos en la cocaína. España es, junto a USA y el Reino Unido, uno de los países del mundo con un uso más extendido de esta droga ${ }^{43,44}$, con una prevalencia en los últimos 12 meses en 2003 de 2,7\% para 1564 años y de $4,8 \%$ para $15-34$ años $^{8}$. Con los datos publicados ${ }^{8}$ se puede estimar que en España hay más de 100.000 consumidores semanales de esta droga. La gran mayoría la usa por vía intranasal (esnifada). Los que se la inyectan o la fuman se concentran entre los usuarios de heroína, sin que hasta ahora se haya detectado un aumento importante de estas formas de uso en la población general. En cualquier caso, la cocaína provoca ya muchos problemas sanitarios, superando claramente a la heroína como causa de primeras admisiones a tratamiento por dependencia o de urgencias hospitalarias ${ }^{8,15,39,45}$. Se estima que en España en 2005 el número de personas admitidas a tratamiento por dependencia de cocaína pudo situarse en torno a $25.000^{8,14,15}$, y que en Barcelona en la población de 15-54 años hay un 3,3\% (IC95\%:1,4\%-7,0\%) de consumidores problemáticos de drogas que usan cocaína ${ }^{46}$.

Todos los indicadores muestran una tendencia creciente del uso y los problemas por cocaína $^{8,45}$ (figura 2). Así, entre 2002 y 2004 las personas admitidas a tratamiento por esta droga pasaron de 1.728 a 3.565 en Andalucía $^{15}$, y de 2.168 a 3.683 en Cataluña ${ }^{14}$. El número de decomisos de cocaína pasó de 925 en 1984 a 4.132 en 1992 y 37.707 en 2005, y la cantidad decomisada de 277 a 4.554 y $48.429 \mathrm{Kg}$, respectivamente ${ }^{8,47,48}$. El aumento de los decomisos no ha servido, sin embargo, para reducir la disponibilidad de cocaína percibida por la población, que ha seguido creciendo (figura 3$)^{8}$.
Los cuadros clínicos en las urgencias relacionadas con cocaína dependen del uso o no de otras drogas. Entre los que no usan opioides, que ahora son mayoría, predominan las complicaciones psiquiátricas (sobre todo crisis de ansiedad y psicosis) u orgánicas (sobre todo taquicardia y dolor torácico), y las intoxicaciones agudas ${ }^{49,51}$. Las complicaciones más graves son los accidentes cerebrovasculares y cardiovasculares, incluyendo arritmias y episodios coronarios agu$\operatorname{dos}^{52}$. También pueden ser frecuentes las lesiones por causas externas. En este sentido, los datos de Itinere muestran que el riesgo de accidentes de los jóvenes usuarios regulares de cocaína es más de dos veces mayor que el de la población general de la misma edad $^{53}$. La presencia de cocaína en las muertes objeto de investigación judicial por reacción aguda a drogas ha aumentado ${ }^{8}$, pero es probable que la mayoría de las muertes con implicación de cocaína no lleguen a ser objeto de investigación judicial y forense y pasen por «muertes naturales» de origen cardiocirculatorio. Además, en muchas muertes accidentales no se hace investigación toxicológica completa, por lo que puede decirse que en España se desconoce el impacto real de la cocaína sobre la mortalidad. Hay que resaltar que los consumidores regulares de cocaína tienen un elevado índice de comorbilidad psiquiátrica: $43,6 \%$ de los jóvenes usuarios de cocaína de Itinere la presentaban; $30,8 \%$ relacionada con eje I de DSM-IV (trastornos del estado de animo, de la alimentación, ansiedad, o psicosis); 5,3\% con eje II (trastornos de la personalidad), y $7,5 \%$ con ambos ejes ${ }^{54}$.

Por lo que respecta al cannabis el consumo ha crecido mucho en los últimos años, pero sus repercusiones sanitarias siguen siendo bastante menores que las de la heroína o la cocaína. En 2003 en España la prevalencia de uso de cannabis en los últimos 12 meses fue del $11,3 \%$ en el grupo de edad 1554 años y del $20,1 \%$ en el de $15-34^{8,55}$. A partir de los datos publicados ${ }^{8,14,15}$ se estima que hay en torno a medio millón de consumido- 
Figura 2

Tendencias del consumo y los problemas causados por cocaína en España. 1994-2004 (Índice 1996=100)

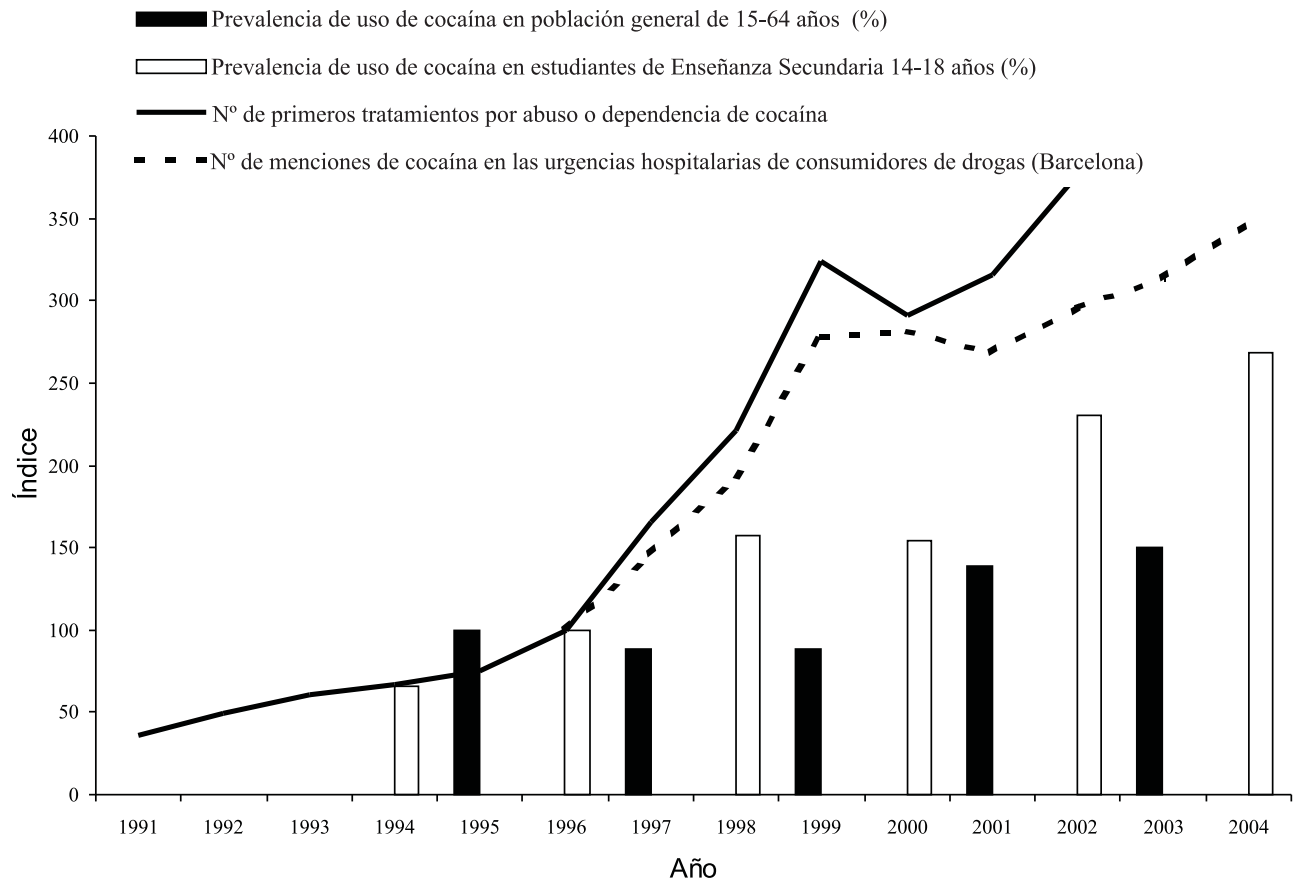

Fuente: La prevalencia de uso de cocaína en población general se ha obtenido de la Encuesta Domiciliaria sobre Alcohol y Drogas en España (EDADES $)^{8}$ y la prevalencia de uso entre los estudiantes de la Encuesta Estatal sobre Uso de Drogas en Enseñanzas Secundarias $\left(\right.$ ESTUDES ${ }^{8 ; 89}$. Los datos de tratamiento proceden del indicador tratamiento del Plan Nacional sobre Drogas $(P N D)^{8,36}$, y los de urgencias del Sistema d'Informació de Drogodependéncies de Barcelona $(\operatorname{SIDB})^{39}$.

res diarios, y que en 2005 fueron admitidas a tratamiento por abuso o dependencia de cannabis 6.500-7.000 personas, la gran mayoría por primera vez en la vida. No hay apenas evidencias sobre los problemas que llevan a estas personas a tratamiento, aunque en muchos casos podrían acudir para evitar las sanciones administrativas derivadas del consumo en lugares públicos. Aparentemente el impacto del uso de cannabis en la atención sanitaria urgente es mayor que en los centros de tratamiento especializado. De hecho en 2002 estaban relacionadas con uso de cannabis aproximadamente el $20 \%$ de las urgencias hospitalarias por reacción aguda a drogas ilegales ${ }^{8}$. Sin embargo, a menudo eran policonsumidores y la urgencia estaba tam- bién relacionada con otras drogas, como alcohol o cocaína. Aunque no se han detectado muertes por reacción aguda a cannabis es probable que algunos accidentes mortales y no mortales se relacionen con esta droga, consumida de forma aislada o en combinación con otras ${ }^{53}$.

Los psicoestimulantes sintéticos tipo éxtasis o anfetaminas tomados en un contexto lúdico o festivo fueron objeto de preocupación desde principios de los noventa ${ }^{56,57}$. Las evidencias muestran que estas drogas pueden producir problemas de salud ${ }^{58}$, en algunos casos graves, pero su volumen no justifica en absoluto que durante varios años coparan muchos discursos y mentideros 
Figura 3

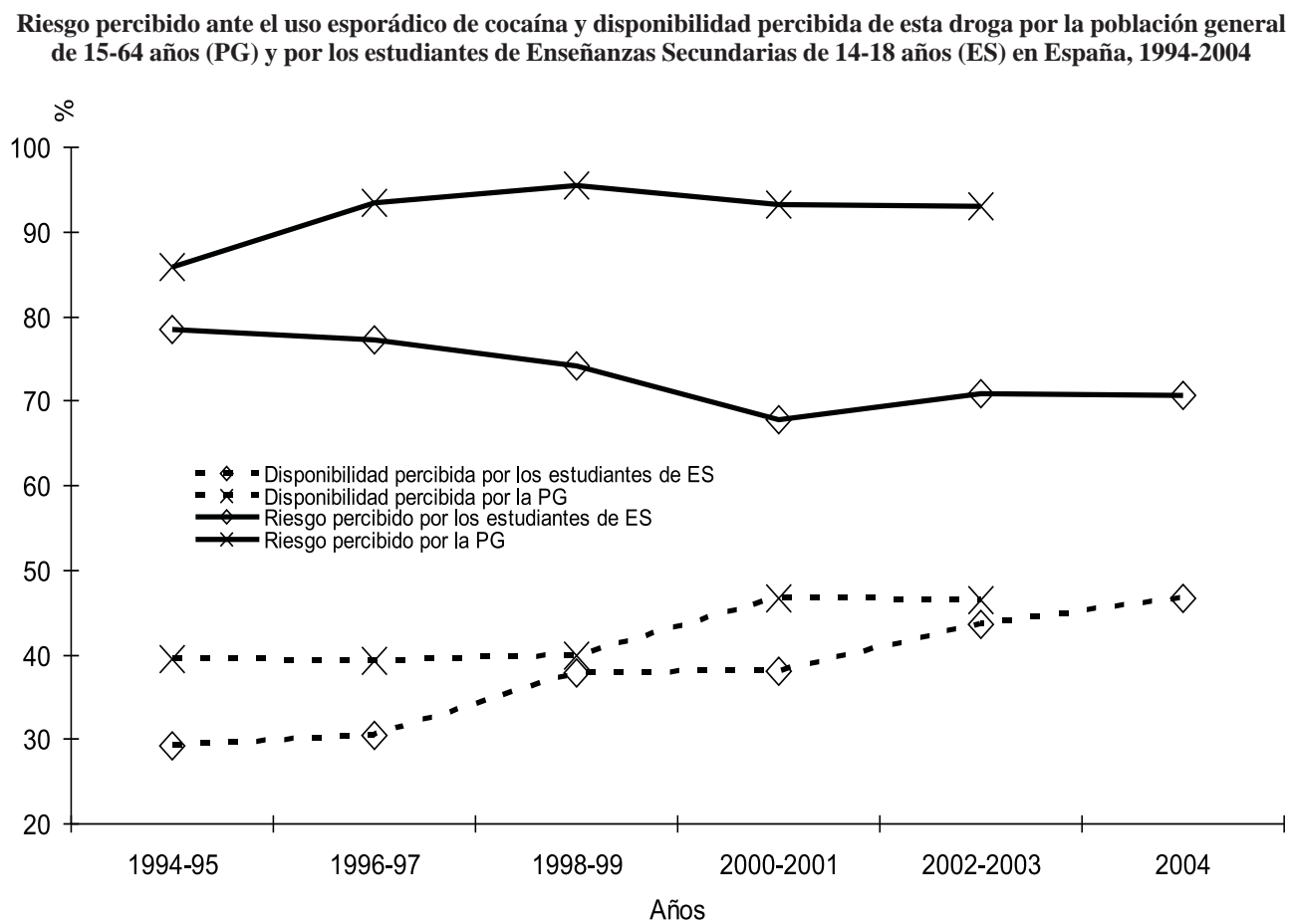

Disponibilidad percibida: Porcentaje de encuestados que considera que podría obtener fácil o muy fácilmente cocaína en 24 horas.

Riesgo percibido: Porcentaje de encuestados que considera que el uso esporádico de cocaína (una vez al mes o menos frecuentemente) puede producir muchos o bastantes problemas.

Fuente: Los datos para la Población General (PG) se han obtenido de la Encuesta Domiciliaria sobre Alcohol y Drogas en España (EDADES) ${ }^{8}$ y para los estudiantes de Enseñanzas Secundarias (ES) de la Encuesta Estatal sobre Uso de Drogas en Enseñanzas Secundarias (ESTUDES $)^{8 ; 89}$.

políticos y profesionales, y llegaran a convertirse aparentemente en una de las máximas prioridades de la política de drogas (por encima de drogas a la postre mucho más problemáticas como la cocaína). De hecho, la mención de estos psicoestimulantes en las urgencias hospitalarias por reacción aguda a drogas se sitúa en los últimos años en torno al $10 \%$ del total, las muertes anuales por intoxicación aguda se cuentan con los dedos de una mano (y dado el contexto en el que se producen, no es probable que pasen desapercibidas), y el número anual de tratamientos por abuso o dependencia no llega a 1.000 (menos del 2\% del total y menos del $5 \%$ de las primeras admisiones) ${ }^{8,15,37,39,59}$. La respuesta al fenómeno del uso de «éxtasis» en España quedará probablemente como un ejemplo de respuesta inspirada más por el ansia de vender o comprar novedades que por problemas o evidencias. Cuando se comenzó a mentar insistentemente este «problema», desde la perspectiva epidemiológica estaba muy claro que la heroína producía la gran mayoría de las consecuencias adversas relacionadas con drogas ilegales, que las iba a seguir produciendo a corto plazo, y que la cocaína se vislumbraba como la gran amenaza. Sin embargo, los intentos de poner esto en evidencia y dudar de la versión 
oficial $^{60}$ se pretendieron silenciar de forma bastante expeditiva.

\section{ALGUNAS PROPUESTAS DESDE LA HISTORIA Y LAS EVIDENCIAS}

Las propuestas que se hacen a continuación parten de las siguientes premisas. La primera es que en las últimas décadas España ha sido uno de los países del mundo donde las consecuencias del uso de drogas ilegales han sido más desastrosas para la salud pública y, sin embargo, las evaluaciones de las políticas públicas sobre drogas han sido y siguen siendo muy autocomplacientes. Así, en la presentación de la Estrategia Nacional sobre Drogas 2000-2008 se dice que con lo hecho desde 1985 «nuestro país puede sentirse razonablemente satisfecho», y que «se han alcanzado una serie de logros que nos colocan entre los países más avanzados en el desarrollo de las políticas frente a las drogas» ${ }^{40}$. Perfecto, si junto a los logros evidentes (desarrollo de una red amplia y diversificada de atención a los consumidores), se hubiese reconocido que el rastro de la epidemia de heroína habría sido menor con respuestas oportunas y basadas en la evidencia, aparcando apriorismos ideológicos, e intereses políticos y profesionales (por ejemplo implantando los TMM 5-8 años antes), y también que no se estaba siendo capaz de contener el aumento del uso de cocaína y cannabis. Aunque afortunadamente en la evaluación 2003 de esa estrategia se reconoció que la prevención del consumo, objetivo prioritario de la misma, no presentaba resultados satisfactorios ${ }^{41,42}$. De hecho, durante los últimos diez años el uso de cocaína y cannabis ha aumentado considerablemente, $y$, aunque son fenómenos (sobre todo el del cannabis) que afectan a buena parte de Europa, no hay que descartar que en España los programas preventivos, los de control de la oferta, o ambos hayan sido insuficientes, inefectivos o hayan estado mal orientados. Por otra parte, aunque las políticas públicas son un determinante importante de la evolu- ción del uso y los problemas ocasionados por las drogas, no hay que sobrevalorar su efecto, porque existen otros factores que pueden contribuir a determinar las tendencias. Así ha ocurrido con el cambio de vía de administración de heroína, de inyectada a fumada, que en España ha sido fundamental para controlar la epidemia de $\mathrm{VIH}^{10,12}$. La segunda premisa es que España no es (ni ha sido) un área homogénea en cuanto a la situación de los problemas relacionados con las drogas ni en las respuestas preventivo-asistenciales. La administración central tiene desde hace años competencias muy limitadas y decrecientes en este tema, concentrándose las mismas en las administraciones autonómica y local, que en algunos casos han desarrollado respuestas tardías y heterogéneas. De hecho, algunas diferencias en los problemas con las drogas (por ejemplo la prevalencia de infección por VIH) podrían achacarse en parte a la heterogeneidad en el tipo o calendario de implantación de las intervenciones (los TMM, por ejemplo) $)^{24,61}$.

Y a continuación las propuestas. La primera es que los usuarios de heroína, sobre todo los que se inyectan, han de seguir siendo objeto de programas intensivos de prevención, reducción del daño y vigilancia epidemiológica. Y hay que insistir en ello porque desde algunos sectores podría cometerse el error de dar por liquidado el problema de la inyección y de la heroína, corriendo el riesgo de disminuir prematuramente la dotación de estos programas. El reciente aumento en Londres de la incidencia de VIH y VHC ligada a la inyección se ha atribuido a la relajación de estos programas ${ }^{62}$. Además, se sabe que «las epidemias» de consumo se producen de forma cíclica y muy ligada a cohortes generacionales: cada droga es de una generación y un momento, tiene su clímax y es sustituida por otra en la siguiente generación, como parte del proceso de diferenciación e innovación generacional. Posteriormente los «viejos hábitos» son retomados por otra generación, que aprende de «los 
abuelos». En nuestra opinión, el mantenimiento y refuerzo de los programas dirigidos a usuarios de heroína se justifica no sólo por razones éticas y humanitarias sino también por razones estrictas de salud pública (padecen a menudo infecciones que pueden transmitirse a la población general, como VIH o hepatitis). Por ejemplo, una reemergencia de la inyección en una nueva generación tendría un impacto sobre la salud pública mucho más fuerte en España que en otros países.

El hallazgo reciente de nuevos casos de infección por VIH en jóvenes usuarios de heroína que no se la inyectan apoya la necesidad de reforzar los programas para evitar la transmisión del virus desde los que sí se la inyectan y son seropositivos a sus parejas sexuales. Hay que reforzar también los programas de vacunación frente al VHB y VHA, usando preferentemente pautas de corta duración para mejorar su cumplimiento (3 dosis-3 semanas para VHB $)^{63,64}$, porque aunque desde 1982 se recomienda vacunar frente a VHB a las personas con riesgo, un estudio reciente muestra que la protección es bastante baja, y que se pierden muchas oportunidades de vacunar en los servicios de atención ${ }^{65}$. A pesar de ello, las perspectivas de control del VHB a medio plazo son optimistas, dado que en 19911995 se inició la vacunación sistemática de los adolescentes consiguiéndose una cobertura cercana al $80 \%$, e incluyéndose más tarde esta vacuna en el calendario vacunal infantil. Más difícil es el control del VHC que pasa necesariamente por evitar la infección y detectar y tratar con antivirales a los sujetos infectados. La prevención del VHC exige el refuerzo de los programas de reducción del daño (metadona, distribución de materiales de inyección estériles, salas de consumo, etc.), insistiendo en evitar el inicio en la inyección (ya que la mayoría se infectan en los dos primeros años de inyección) y en evitar compartir además de las agujas y jeringas otros materiales para inyectarse o preparar la droga (recipientes, filtros, líqui- dos, etc.) o utilizar droga preparada en jeringas usadas ${ }^{66,67}$. Además, en las prisiones, donde se concentran mucho las personas que se inyectan, hay que ampliar y mejorar el acceso a material de inyección estéril, porque hay evidencias de que su desinfección con lejía no es efectiva ${ }^{67-69}$, y probablemente el funcionamiento de los PIJs en algunas prisiones españolas es muy limitado. Con respecto al tratamiento del VHC se sabe que en algunos lugares se establecen múltiples contraindicaciones, a menudo con poco apoyo empírico, que excluyen del tratamiento a muchos inyectores de drogas. Hay que insistir en que la inyección o el uso de drogas no es en sí mismo una contraindicación, ni los cuadros depresivos, y tampoco está claro que lo sea el uso moderado de alcohol ${ }^{70}$. Por tanto ha de valorarse su indicación en cada caso individual y facilitar al paciente el cumplimiento del tratamiento ${ }^{71}$. En este sentido, incluir a los afectados en TMM y desarrollar protocolos de actuación consensuados entre las administraciones, que prevean una buena coordinación entre los servicios sanitarios generales (hospitalarios y extrahospitalarios) y los de prisiones (donde se concentran muchos infectados) puede facilitar el cumplimiento $^{33,72,73}$.

Por otra parte, dado que las muertes por «sobredosis» son en gran medida evitables y que siguen siendo frecuentes en España, es muy preocupante que su prevención no aparezca generalmente como un objetivo o prioridad explícita de las estrategias, planes de acción ${ }^{40,42}$ o discursos políticos, aunque se incluye tímidamente en algunos planes, como el de Barcelona o el País Vasco ${ }^{74,75}$. Algunos países con menos problemas que España han incluido explícitamente este objetivo en sus estrategias públicas y han desarrollado programas de entrenamiento de los consumidores para evitar las sobredosis y sus consecuencias, distribución de naloxona y coordinación de servicios para disminuir el riesgo en períodos críticos (por ejemplo, al salir de prisión ${ }^{76-79}$. En esta línea, el Consejo Europeo recomienda a los gobier- 
nos desarrollar políticas para reducir estas muertes, ya que son una de las principales causas evitables de mortalidad juvenil y están en niveles históricamente altos ${ }^{80}$.

Aunque es más razonable centrar las estrategias en problemas que en drogas concretas, para reducir el riesgo de que en España pueda reproducirse el panorama vivido con la heroína hay que aumentar sustancialmente los recursos y los programas destinados a frenar los problemas por cocaína. Puede ser recomendable desarrollar un plan de acción global entre las administraciones que valore los recursos disponibles y prevea intervenciones para reducir el uso y las consecuencias de esta droga (problemas orgánicos y psicopatológicos agudos, accidentes, prácticas sexuales con riesgo de transmisión de infecciones, etc.). Deben establecerse igualmente estrategias razonables de control de la oferta. En este sentido, las encuestas ${ }^{8}$ (figuras 2 y 3 ) sugieren que las incautaciones apenas tienen efecto sobre la creciente facilidad de acceso de la población a cocaína. No es fácil enfocar correctamente las acciones de control de la oferta. De hecho, los contundentes programas de Estados Unidos en este campo tampoco han arrojado resultados espectaculares, y seguramente sí bastantes efectos adversos. En cuanto a los programas de prevención del consumo habría que evaluar su dimensión y efectividad, reforzando la prevención selectiva e indicada, porque la estrategia seguida hasta ahora (basada sobre todo en la prevención universal y en campañas más o menos ingeniosas en los medios de comunicación) no ha logrado detener el aumento del consumo, a pesar de que el riesgo percibido ante el mismo por la población general y juvenil se ha mantenido estabilizado en niveles altos. En este sentido, algunos estudios muestran que las intervenciones breves basadas en la motivación en un contexto clínico pueden reducir el consumo de cocaína o heroína, así como los episodios de urgencias relacionados con las drogas ${ }^{81-83}$. En cualquier caso, las revisiones más recientes sugieren que, aunque hay ciertas eviden- cias de que las intervenciones basadas en las habilidades pueden ser efectivas en el medio escolar y las basadas en la motivación pueden serlo en otros ámbitos, en general, estas evidencias son débiles ${ }^{84,85}$, por lo que cualquier apuesta en este terreno sigue siendo arriesgada.

Los profesionales sanitarios de los servicios de salud (sobre todo los de atención primaria de salud y servicios de urgencias) han de preguntar sistemáticamente por el uso de cocaína o cannabis a todas las personas con cuadros que pudieran estar relacionados con dichas drogas, especialmente a las personas de entre 15 y 54 años con problemas psicopatológicos, cardiocirculatorios o neurológicos agudos. Además, como muchos consumidores no son conscientes de su problema deben mejorar su capacidad para detectar la dependencia aplicando escalas muy breves como la Severity Dependence Scale $(\mathrm{SDS})^{86,87}$, y derivando a centros especializados a las personas que sobrepasen un cierto umbral de puntuación. Finalmente, deben realizar consejo o intervenciones breves basadas en la motivación a todos los consumidores, incluidos los que no cumplen criterios de abuso o dependencia, porque se ha comprobado que pueden contribuir a reducir el consumo de drogas ${ }^{81-83}$.

Los centros especializados en tratamiento de drogodependencias han de estar bien dotados para ofrecer programas atractivos a los usuarios de cocaína y cannabis, y facilitar siempre una adecuada evaluación psicopatológica, ya que en la población consumidora de estas sustancias son muy frecuentes los problemas de salud mental ${ }^{54}$.

Finalmente, hay que emprender de forma urgente investigaciones para evaluar el impacto de la cocaína sobre la mortalidad y accidentabilidad, así como programas de formación de los médicos para reconocer y certificar adecuadamente las muertes relacionadas con el uso de esta droga. 


\section{BIBLIOGRAFÍA}

1. Barrio G, de la Fuente L, Camí J. El consumo de drogas en España y su posición en el contexto europeo. Med Clin (Barc) 1993;101:344-55.

2. Sánchez Niubó A, Domingo-Salvany A, Gómez, J, Brugal MT. Estimación de la incidencia de consumo de drogas. Gac Sanit 2005; 19(Supl 1): 98.

3. de la Fuente L, Barrio G, Vicente J, Bravo MJ, Santacreu J. The impact of drug-related deaths on mortality among young adults in Madrid. Am J Public Health $1995 ; 85(1): 102-5$.

4. Montellà C, Borrell C, Brugal MT, Plasència A. Evolución de la mortalidad en los jóvenes de la ciudad de Barcelona: 1983-1993. Med Clin (Barc) 1997;108:241-7.

5. Ortí RM, Domingo-Salvany A, Muñoz A, Macfarlane D, Suelves JM, Antó JM. Mortality trends in a cohort of opiate addicts, Catalonia, Spain. Int J Epidemiol 1996;25:545-53.

6. Brugal MT, Barrio G, Regidor E, Mestres M, Caylà $\mathrm{JA}$, de la Fuente L. Discrepancias en el número de muertes por reacción aguda a sustancias psicoactivas registradas en España. Gac Sanit 1999; $13: 82-7$

7. Brugal MT, Barrio G, Royuela L, Bravo MJ, de la Fuente L, Regidor E. Estimación de la mortalidad atribuible al consumo de drogas ilegales en España. Med Clin (Barc) 2004;123:775-7.

8. Delegación del Gobierno para el Plan Nacional sobre Drogas. Observatorio Español sobre Drogas (OED). Informe 2004. Madrid: Ministerio de Sanidad y Consumo; 2005.

9. Castilla J,.de la Fuente L. Evolución del número de personas infectadas por el virus de la inmunodeficiencia humana y de los casos de sida en España: 1980-1998. Med Clin (Barc) 2000;115:85-9.

10. de la Fuente L., Bravo MJ, Barrio G, Parras F, Suárez M, Rodés A et al. Lessons from the history of the human immunodeficiency virus/acquired immunodeficiency syndrome epidemic among Spanish drug injectors. Clin Infect Dis 2003;37 Suppl 5:S410-S415.

11. de la Fuente L, Barrio G, Royuela L, Bravo MJ. The transition from injecting to smoking heroin in three Spanish cities. The Spanish Group for the Study of the Route of Heroin Administration. Addiction. 1997;92:1749-63.
12. de la Fuente L, Barrio G, Bravo MJ, Royuela L. Heroin smoking by «chasing the dragon»: its evolution in Spain. Addiction. 1998;93:444-6.

13. de la Fuente L, Saavedra P, Barrio G, Royuela L, Vicente J, Spanish Group for the study of the purity of seized drugs. Temporal and geographic variations in the characteristics of heroin seized in Spain and their relation with the route of administration. Drug Alcohol Depend 1996;40:185-94.

14. Òrgan Tècnic de Drogodependències. Sistema d'Informació sobre drogodependéncies a Catalunya. Informe anual 2004. 2005. Barcelona; Generalitat de Catalunya.

15. Dirección General para las Drogodependencias y Adicciones. Observatorio Andaluz sobre Drogas y Adicciones. Informe sobre el indicador admisiones a tratamiento en Andalucía en 2005. 2006. Sevilla: Junta de Andalucía. Consejería para la Igualdad y el Bienestar Social; 2006.

16. Ministerio de Sanidad y Consumo. Gabinete de Prensa. Encuesta Estatal sobre Salud y Drogas entre los Internados en Prisión (ESDP), 2006. Madrid: Ministerio de Sanidad y Consumo; 2006.

17. Brugal MT, Domingo-Salvany A, Puig R, Barrio G, García de Olalla P, de la Fuente L. Evaluating the impact of methadone maintenance programmes on mortality due to overdose and aids in a cohort of heroin users in Spain. Addiction. 2005;100:981-9.

18. Rodríguez-Llera MC, Domingo-Salvany A, Brugal MT, Silva TC, Sánchez-Niubó A, Torrens M. Psychiatric comorbidity in young heroin users. Drug Alcohol Depend 2006;84:48-55.

19. Caballero L. Adicción a la cocaína: neurobiología, clínica, diagnóstico y tratamiento. Madrid: Ministerio de Sanidad y Consumo; 2005.

20. López-Gay D, Peña-Rey I, Izarra C, Monteagudo O. Vigilancia epidemiológica de la infección por VIH/Sida en la Comunidad de Madrid, hasta diciembre de 2003. Bol Epidemiol de la Comunidad de Madrid 2004;10:3-88.

21. Centro Nacional de Epidemiología. Instituto de Salud Carlos III. Proyecto EPI-VIH. Evolución de la prevalencia de VIH en pacientes de once centros de enfermedades de transmisión sexual y/o de diagnóstico del VIH, 1991-2004. Madrid: Ministerio de Sanidad y Consumo; 2006. Disponible en: http:// cne.isciii.es/htdocs/sida/evolucion.pdf . 20-62006. [citado 20-6-2006] .

22. Centro Nacional de Epidemiología. Instituto de Salud Carlos III. Sistema de Información sobre 
nuevos diagnósticos de VIH Autonómicos. Vigilancia epidemiológica de VIH en España. Valoración de la epidemia de VIH en España a partir de los sistemas de notificación de casos de las comunidades autónomas. Actualización año 2005. Disponible en: http://www.isciii.es/htdocs/pdf/nuevos_diagnosticos_ccaa.pdf. [citado 20-6-2006] .

23. Vallejo F, Toro C, Brugal MT, de la Fuente, Soriano V, Jiménez R, Ballesta R, and Bravo MJ. Muy alta incidencia de VIH y VHC en jóvenes consumidores de heroína. 2006. XXIV Reunión Científica Anual de la Sociedad Española de Epidemiología. Logroño 3-10-2006.

24. de la Fuente L, Bravo MJ, Toro C, Brugal MT, Barrio G, Soriano V et al. Injecting and HIV prevalence among young heroin users in three Spanish cities and their association with the delayed implementation of harm reduction programmes. J Epidemiol Community Health 2006;60:537-42.

25. Bravo MJ, Lacasa D, Silva TC, Vallejo F, Martínez G, Toro C et al. Prevalencia de infección por VHB en jóvenes consumidores de heroína (inyectores y no inyectores). Gac Sanit 2005;19 (Supl 1):49.

26. Bassani S, Toro C, de la FL, Brugal MT, Jiménez V, Soriano V. Prevalencia de infección por virus de transmisión parenteral en consumidores actuales de heroína de 3 ciudades españolas. Med Clin (Barc) 2004;122:570-2.

27. Wiessing L, Roy K, Sapinho D, Hay G, Taylor A, Goldberg D et al. Surveillance of hepatitis C infection among injecting drug users in the European Union. In Jager J, Limburg W, Kretzschmar M, Postma M, Wiessing L, eds. Hepatitis $\mathrm{C}$ and injecting drug use: impact, costs and policy options, pp 21-38. Luxembourg: Office for Official Publications of the European Communities; 2004.

28. Muga R, Sanvisens A, Bolao F, Tor J, Santesmases J, Pujol R et al. Significant reductions of HIV prevalence but not of hepatitis $\mathrm{C}$ virus infections in injection drug users from metropolitan Barcelona: 1987-2001. Drug Alcohol Depend 2006;82 Suppl 1:S29-S33.

29. Soriano V, Martín-Carbonero L, García-Samaniego J, Puoti M. Mortality due to chronic viral liver disease among patients infected with human immunodeficiency virus. Clin Infect.Dis 2001;33:1793-5.

30. Lumbreras B, Jarrin I, Del Amo J, Pérez-Hoyos S, Muga R, García-de la Hera M et al. Impact of hepatitis $\mathrm{C}$ infection on long-term mortality of injecting drug users from 1990 to 2002: differences before and after HAART. AIDS 2006;20:111-6.
31. Vallet-Pichard A, Pol S. Natural history and predictors of severity of chronic hepatitis $\mathrm{C}$ virus (HCV) and human immunodeficiency virus (HIV) coinfection. J Hepatol. 2006;44:S28-S34.

32. Brugal MT, Frigola M, Vallejo F, Ruiz S, Ambrós M, Bravo MJ et al. Prevalencia de hepatitis C y coinfección con VIH entre los jóvenes inyectores de drogas. Gac Sanit 2005;19 (Supl 1):111.

33. Bruguera M, Forns X. Hepatitis C en España. Med Clin (Barc) 2006;127:113-7.

34. Bravo MJ, Barrio G, de la Fuente L, Colomo C, Royuela L, Grupo de Trabajo de Médicos del Mundo. Persistencia de conductas de riesgo para la transmisión del VIH en inyectores de drogas de Madrid, Sevilla y Valencia. Gac Sanit 1999;13: 109-18.

35. Bravo MJ, Royuela L, Barrio G, Rodríguez-Arenas MA, de la Fuente L. Prevalencia de prácticas indirectas de compartir material para inyectarse drogas en Galicia, Madrid, Sevilla y Valencia. Gac Sanit 2004;18:472-8.

36. Delegación del Gobierno para el Plan Nacional sobre Drogas. Observatorio Español. Plan Nacional sobre Drogas. Indicadores tratamiento, urgencias y mortalidad. Informe año 2002. Madrid: Ministerio de Sanidad y Consumo; 2006. Disponible en: http://www.pnsd.msc.es/Categoria2/obser$\mathrm{va} / \mathrm{seipad} /$ indicad_2002.htm [citado 21-06-06].

37. Dirección General para las Drogodependencias y Adicciones. Observatorio Andaluz sobre Drogas y Adicciones. Informe sobre el indicador mortalidad por reacción aguda a sustancias psicoactivas. 2004. Sevilla, Junta de Andalucía. Consejería para la Igualdad y el Bienestar Social; 2006.

38. Agencia Antidroga de la Comunidad de Madrid. Evolución de la mortalidad. Madrid: Consejería de Sanidad y Consumo de la Comunidad de Madrid; 2004. Disponible en: http://www.madrid.org/ web_agencia_antidroga/indicadores/ffindicadores.htm [Citado el 21-4-2004].

39. Brugal MT, Queralt, A, Graugés, D, García V, Vecino C. Sistema d'Informació de Drogodependències de Barcelona. Actualització dels indicadors de drogues corresponent al 2n trimestre del 2005. Barcelona: Agència de Salut Pública de Barcelona; 2005 ..

40. Delegación del Gobierno para el Plan Nacional sobre Drogas. Estrategia nacional sobre drogas, 2000-2008. Madrid: Ministerio del Interior;2000. 
41. Delegación del Gobierno para el Plan Nacional sobre Drogas. Evaluación 2003. Estrategia nacional sobre drogas, 2000-2008. Madrid: Ministerio de Sanidad y Consumo; 2005.

42. Delegación del Gobierno para el Plan Nacional sobre Drogas. Plan de Acción 2005-2008. Estrategia nacional sobre drogas, 2000-2008. Madrid: Ministerio de Sanidad y Consumo;2005.

43. United Nations Office on Drug and Crime (UNODC). 2006 World drug report. Vienna: UNODC; 2006.

44. Observatorio Europeo de las Drogas y las Toxicomanías. Informe anual 2005. El problema de la drogodependencia en Europa. Luxemburgo: Oficina de Publicaciones Oficiales de las Comunidades Europeas; 2005.

45. Suelves JM, Brugal MT, Cayla JA, Torralba L. Cambio de los problemas de salud provocados por la cocaína en Cataluña. Med Clin.(Barc.) 2001;117:581-3.

46. Brugal MT, Domingo-Salvany A, Díaz E, Torralba L. Prevalence of problematic cocaine consumption in a city of southern Europe, using capture-recapture with a single list. J Urban Health 2004;81:41627.

47. Delegación del Gobierno para el Plan Nacional sobre Drogas. Informe $n^{\circ} 1$. Observatorio Español sobre Drogas. Madrid: Ministerio del Interior; 1998.

48. Ministerio del Interior. Secretaría de Estado de Seguridad. Estadística anual sobre drogas. 2005. Madrid: Ministerio del interior; 2006.

49. Barrio G, Rodríguez-Arenas MA, de La Fuente L, Royuela L, Grupo de Trabajo para el Estudio de las Urgencias por Psicoestimulantes. Urgencias en consumidores de cocaína en varios hospitales españoles: primeras evidencias de complicaciones agudas por consumo de crack. Med Clin (Barc) 1998;111:49-55.

50. Brugal MT. La cocaína a Barcelona. Característiques i problemes dels consumidors detectats als serveis d'urgencies. Una estimació de la prevalença. 2000. Barcelona: 23-6-2006.

51. Sanjurjo E, Montori E, Nogué S, Sánchez M, Munné P. Urgencias por cocaína: un problema emergente. Med Clin (Barc) 2006;126:616-9.

52. Muga R. Y ahora la cocaína. Med Clin (Barc) 2001;117:584-5.
53. Sánchez F, Pulido J, Barrio G, Ballesta R, Rodríguez A, Castellano Y, Domingo-Salvany A, Regidor E. Accidentes entre los jóvenes consumidores de cocaína: prevalencia, circunstancias y factores asociados. XXIV Reunión Científica Anual de la Sociedad Española de Epidemiología. 2006. Logroño; 3-10-2006.

54. Herrero MJ, Domingo-Salvany A, Brugal MT, Lacasa D, Investigadores ITINERE. Comorbilidad psiquiátrica en consumidores jóvenes de cocaína. 2006. XXIV Reunión Científica Anual de la Sociedad Española de Epidemiología. Logroño; 3-102006.

55. Delegación del Gobierno para el Plan Nacional sobre Drogas. Informe sobre cannabis. Comisión Clínica. Madrid: Ministerio de Sanidad y Consumo; 2006.

56. de la Fuente L, Rodríguez-Arenas MA, Vicente J, Sánchez J, Barrio G. Epidemiología del consumo de drogas de diseño en España. Med Clin (Barc) 1997;108:54-61.

57. Gamella J, Álvarez A. Drogas de síntesis en España. Patrones y tendencias de adquisición y consumo. Madrid: Delegación del Gobierno para el Plan Nacional sobre Drogas. Ministerio del Interior; 1997.

58. Rodríguez-Arenas MA, Barrio G, de la Fuente L, Royuela L, Grupo de Trabajo para el Estudio de las Urgencias por Psicoestimulantes. Urgencias relacionadas con el consumo de drogas de diseño, alucinógenos y anfetaminas atendidas en 15 hospitales españoles durante 1994. Rev Clín Esp 1997; 197:804-9.

59. Dirección General para las Drogodependencias y Adicciones. Observatorio Andaluz sobre Drogas y Adicciones. Informe sobre el indicador urgencias relacionadas con el consumo de sustancias psicoactivas en Andalucía. 2004. Sevilla: Junta de Andalucía. Consejería para la Igualdad y el Bienestar Social; 2006.

60. de la Fuente L, Barrio G. Control de los problemas de salud asociados al consumo de drogas en España: Hacia un abordaje científico y priorizado. Gac Sanit 1996;10:255-60.

61. Domingo-Salvany A, Pérez K, Torrens M, Bravo MJ, Antó JM, Alonso J. Methadone treatment in Spain, 1994. Drug Alcohol Depend 1999;56:61-6.

62. Judd A, Hickman M, Jones S, McDonald T, Parry JV, Stimson GV et al. Incidence of hepatitis C virus and HIV among new injecting drug users in 
London: prospective cohort study. BMJ 2005; 330:24-5.

63. Christensen PB, Fisker N, Krarup HB, Liebert E, Jaroslavtsev N, Christensen K et al. Hepatitis B vaccination in prison with a 3 -week schedule is more efficient than the standard 6-month schedule. Vaccine 2004;22:3897-901.

64. Centers for Disease Control and Prevention. Prevention and control of infections with hepatitis viruses in correctional settings. MMWR 2003; 52(RR-1).

65. de la Fuente L, Bravo MJ, Martínez C, Toro C, Lacasa D, Jiménez $\mathrm{F}$ et al. Vacunación frente a VHB en jóvenes consumidores de heroína: prevalencia, factores asociados y oportunidades perdidas. Gac Sanit 2005;19 (Supl 1):92.

66. Organización Panamericana de la Salud (OPS) and Organización Mundial de la Salud (OMS). Encuestas de comportamiento en CODAR. Herramientas básicas para la vigilancia de segunda generación de la transmisión del VIH y otras infecciones en consumidores de drogas con alto riesgo. Documento provisional. Versión 1b-Marzo 2005. 2005. Washington DC: Organización Panamericana de la Salud (OPS); 14-4-2005.

67. Bravo, M. J. Infecciones de transmisión sanguínea o sexual entre las personas que se inyectan drogas y sus parejas en las Américas: manual para profesionales de la salud. Washington DC: Organización Panamericana de la Salud; 2004.

68. Centers for Disease Control and Prevention. Desinfección de jeringuillas para usuarios de drogas inyectables. HIV/AIDS Prevention Bulletin . 2004 (Julio 2004): 1-6.

69. World Health Organization (WHO). Effectiviness of sterile needle and syringe programming in reducing HIV/AIDS among injecting drug users (Evidence for actiontechnical papers). Geneva: WHO 2004.

70. Hagan H, Latka MH, Campbell JV, Golub ET, Garfein RS, Thomas DA et al. Eligibility for treatment of hepatitis $\mathrm{C}$ virus infection among young injection drug users in 3 US cities. Clin Infect.Dis 2006;42:669-72.

71. Kresina TF, Khalsa J, Cesari H, Francis H. Hepatitis $\mathrm{C}$ virus infection and substance abuse: medical management and developing models of integrated care-an introduction. Clin Infect.Dis 2005;40 Suppl 5:S259-S262.
72. Portilla J,.Panel de Expertos GEISIDA-SESP. Protocolo de coordinación para el tratamiento de la hepatitis C crónica en el medio penitenciario. Revista Española de Sanidad Penitenciaria 2004;6:106-12.

73. Soriano V, Miró JM, García-Samaniego J, TorreCisneros J, Núñez M, del Romero J et al. Consensus conference on chronic viral hepatitis and HIV infection: updated Spanish recommendations. J Viral Hepat. 2004;11:2-17.

74. Gobierno Vasco. Dirección de Drogodependencias. V Plan de Drogodependencias de la Comunidad Autónoma de Euskadi; 2004-2008. Vitoria-Gasteiz: Departamento de Publicaciones del Gobierno Vasco; 2006.

75. Ajuntament de Barcelona. Plan d'acció de Drogodepèndencies de Barcelona 2006-2008. Barcelona: Ajuntament de Barcelona; 2006.

76. The Advisory Council on the Misuse of Drugs. Reducing drug-related death. Guidance for drug treatment providers. London: The Stationery Office; 2000.

77. Ministerial Council Drug Strategy. National heroin overdose strategy. Camberra: Commonwealth of Australia. Department of Health and Age Care; 2001.

78. Strategic choices for reducing overdose deaths in four European cities. A joint project of the cities Oslo, Amsterdam, Copenhagen, and Frankfurt am Main. Oslo: Alcohol and Drug Addiction Service; 2002.

79. National Treatment Agency for Substance Misuse (NTA). Reducing drug-related death. Guidance for drug treatment providers. London: NTA; 2004.

80. Hedrich D, Vicente J. Sobredosis: una de las principales causas de mortalidad evitable en jóvenes. Drogas en el punto de Mira. 2004. Lisboa, Observatorio Europeo de Drogas y Toxicomanías (OEDT).

81. Bernstein J, Bernstein E, Tassiopoulos K, Heeren $\mathrm{T}$, Levenson S, Hingson R. Brief motivational intervention at a clinic visit reduces cocaine and heroin use. Drug Alcohol Depend 2005;77:49-59.

82. Tait RJ, Hulse GK. Adolescent substance use and hospital presentations: a record linkage assessment of 12-month outcomes. Drug Alcohol Depend 2005;79:365-71. 
83. Tait RJ, Hulse GK, Robertson SI, Sprivulis PC. Emergency department-based intervention with adolescent substance users: 12-month outcomes. Drug Alcohol Depend 2005;79:359-63.

84. Faggiano F, Vigna-Taglianti FD, Versino E, Zambon A, Borraccino A, Lemma P. School-based prevention for illicit drugs' use. Cochrane .Database Syst Rev 2005;CD003020.

85. Gates S, McCambridge J, Smith LA, Foxcroft DR. Interventions for prevention of drug use by young people delivered in non-school settings. Cochrane Database Syst Rev 2006;CD005030.

86. Kaye $S$, Darke S. Determining a diagnostic cut-off on the Severity of Dependence Scale (SDS) for cocaine dependence. Addiction. 2002;97:727-31.
87. Martin G, Copeland J, Gates P, Gilmour S. The Severity of Dependence Scale (SDS) in an adolescent population of cannabis users: reliability, validity and diagnostic cut-off. Drug Alcohol Depend 2006;83:90-3.

88. Centro Nacional de Epidemiología. Instituto de Salud Carlos III. Vigilancia epidemiológica del Sida en España. Registro Nacional de Casos de Sida. Informe semestral n ${ }^{\circ} 2$. Año 2005. Disponible en: http://www.isciii.es/htdocs/pdf/informe_sida. pdf . 21-6-2006. Actualización a 31 de diciembre de 2005 .

89. Delegación del Gobierno para el Plan Nacional sobre Drogas. Encuesta sobre drogas a la población escolar 1994. Madrid: Ministerio de Justicia e Interior; 1995. 